\title{
Proceeding
}

10th INSHS International Christmas Sport Scientific Conference, 4-5 December 2015. International Network of Sport and Health

Science. Szombathely, Hungary

\section{Accelerometry in sport}

\author{
MILOŠ LUKÁŠEK ${ }^{1}$, RENÁTA VYCHODILOVÁ2 \\ ${ }^{1}$ Department of Athletics, Swimming and Outdoor Sports, Masaryk University, Brno, Czech Republic \\ 2 University Sports Centre Department, Masaryk University, Brno, Czech Republic
}

\begin{abstract}
The possibilities to analyse various sports movements and activities, their techniques and dynamics are deepening and enhancing with the rapid development of modern technologies. It subsequently enables to improve and enhance sports techniques, performances and efficiency, to prevent possible injuries, to enhance training methods, and to reach sports results of higher quality. The use of accelerometry to observe, examine and analyse various movements and their techniques and dynamics in different human physical and sports activities has become of high prominence in several recent decades. The goal of the paper is to illustrate some possibilities of the use of accelerometry to analyse these aspects in the field of sport, in selected cyclic and acyclic sports. The study aims to outline and indicate a way of possible examination, observation and analysis of the data acquired by accelerometry measurements. Key words: MEASUREMENT METHODS, MOVEMENT ACTIVITIES, TECHNIQUE ANALYSIS.
\end{abstract}

Corresponding author. University Sports Centre Department, Masaryk University, Brno, Czech Republic

E-mail: rvychodilova@email.cz

10th INSHS International Christmas Sport Scientific Conference, 4-5 December 2015. International Network of Sport and Health Science. Szombathely, Hungary.

JOURNAL OF HUMAN SPORT \& EXERCISE ISSN 1988-5202

(c) Faculty of Education. University of Alicante

doi:10.14198/jhse.2016.11.Proc1.03 


\section{INTRODUCTION}

Sports performances are determined by different fitness, technical, psychological and anthropometric factors. The use of accelerometry as a research method for the analysis of techniques and dynamics of various types of the human movement and sports activities has increased in recent decades. The use of the method appears to be optimal and convenient in the field research, where the use of other methods seems to be quite difficult, complicated and time consuming. The aim of the paper is to illustrate the possibilities of this measuring technology for its wider utilization in the field of varying movement activities and sports.

The use of accelerometers to analyze the human locomotion is becoming of high prominence among experts nowadays. Accelerometry was first used to analyse basic body movements in medical research (Veltink, et al., 1996). Only later was the method involved to research in the sports environment. In 2002, the results of accelerometry were compared to the ones obtained by measuring with the optoelectronic systems (Mac Reflex system, Qualisys AB) in Sweden. The angular changes of the arms at different velocities were examined (Bernmark and Wiktorin, 2002). This comparison, as well as other comparison where the results of accelometry measurements were compared to the results measured by the kinematic analysis based on the optical principle of the data obtaining (Mayagoitia, et al., 2002), provided the results and conclusions which support the view that the method of accelerometry as highly accurate and precise for field measurements in both the clinical and sports practice.

The subsequent research focused on the use of accelerometers to analyse the dynamic characteristics of the movement. Bogert, et al. (2002) researched the use of accelerometers in the field of the inverse dynamics. Elvin and Arnoczky (2007) examined the reliability of accelerometer in comparison with the dynamic platform. In jumping, the results measured in 30 tested people by the accelerometer and power platform by Bampouras, et al. (2013) showed a high level of correlation. Other research studies in various cyclic movement activities have been carried out, such as walking (Kavanagh, et al., 2008), running (Mizrahi, et al., 2000), and swimming (Nakashima, et al., 2010, and Stamm, et al., 2011). Subsequently, acyclic movement activities have been examined by accelerometers, such as jumping (Sell, et al., 2014), shot putting (Songa, et al., 2006), discus throwing (Liu, et al., 2011) and heading in football (Naunheim, et al., 2003).

\section{MATERIAL AND METHODS}

The accelerometer used in our research was designed and constructed by the author within the internal grant of Masaryk University ("Specific research, MUNI / A / 1000/2011") at the Faculty of Sports Studies, Masaryk University, Brno in 2012. The accelerometer, which was registered by the Industrial Property Office as "the utility model" in 2014, is capable of measuring the linear acceleration and angular velocity in three axes, $x, y$ and $z$.

\section{Hardware}

The device consists of the printed circuit board on which the sensor MPU-6000 containing the three-axial accelerometer and gyroscope are placed. The sensor is set to the maximum range of $\pm 16 \mathrm{~g}$ and $\pm 2000 \% \mathrm{~s}$ in each axis. The sampling frequency is set to $1000 \mathrm{~Hz}$. The raw data is downloaded to the micro SD memory card. The capacity of the card (2GB) enables to conduct measuring for up to 70 hours. The size of the device is $60 \times 38 \times 16 \mathrm{~mm}$, and it is powered by 3 batteries (fig. 1). 


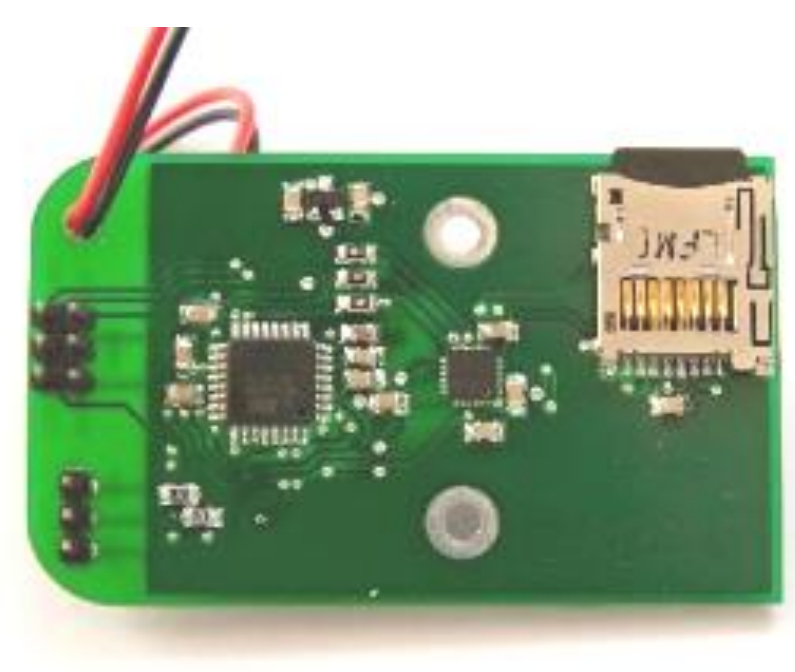

Figure 1. Accelerometer and gyroscope used for measuring

\section{Software}

The programme "SD Card Downlader" was developed at the University of Technology, Brno. The programme is set to reset interferences of the sensor in rest position. The interference values in all axes were measured at the Institute of Physics, the Faculty of Science, Masaryk University, Brno. The program "Next View 4.6 Analyse" was employed to process the data and calculations.

\section{Measuring Procedure}

The accelerometer was placed on a tested person or selected sports tool. It was fixed firmly so as not to move when the sportsperson performed their movement. When the sports performance was completed, the device was turned off and the data was downloaded to the computer. Non-filtered data was used to calculate, filtered data was used to design graphs. To filter the data, the programme "Next View 4.6", low-pass filter, second-rank throughput, cut-off frequency of $50 \mathrm{~Hz}$, filter type Butterworth, was employed.

\section{RESULTS}

\section{Football}

The tested person was a boy aged 11 , a football player attending a specific sports grammar school. The experiment involved heading a football ball flying from the height of $0.5,1.0$ and $1.5 \mathrm{~m}$ from above the player's head. The accelerometer was firmly fixed on the player's occiput with an inflexible band so as not to move. The minus values represent the backward movement of the head after heading the ball on the forehead (fig. 2). 


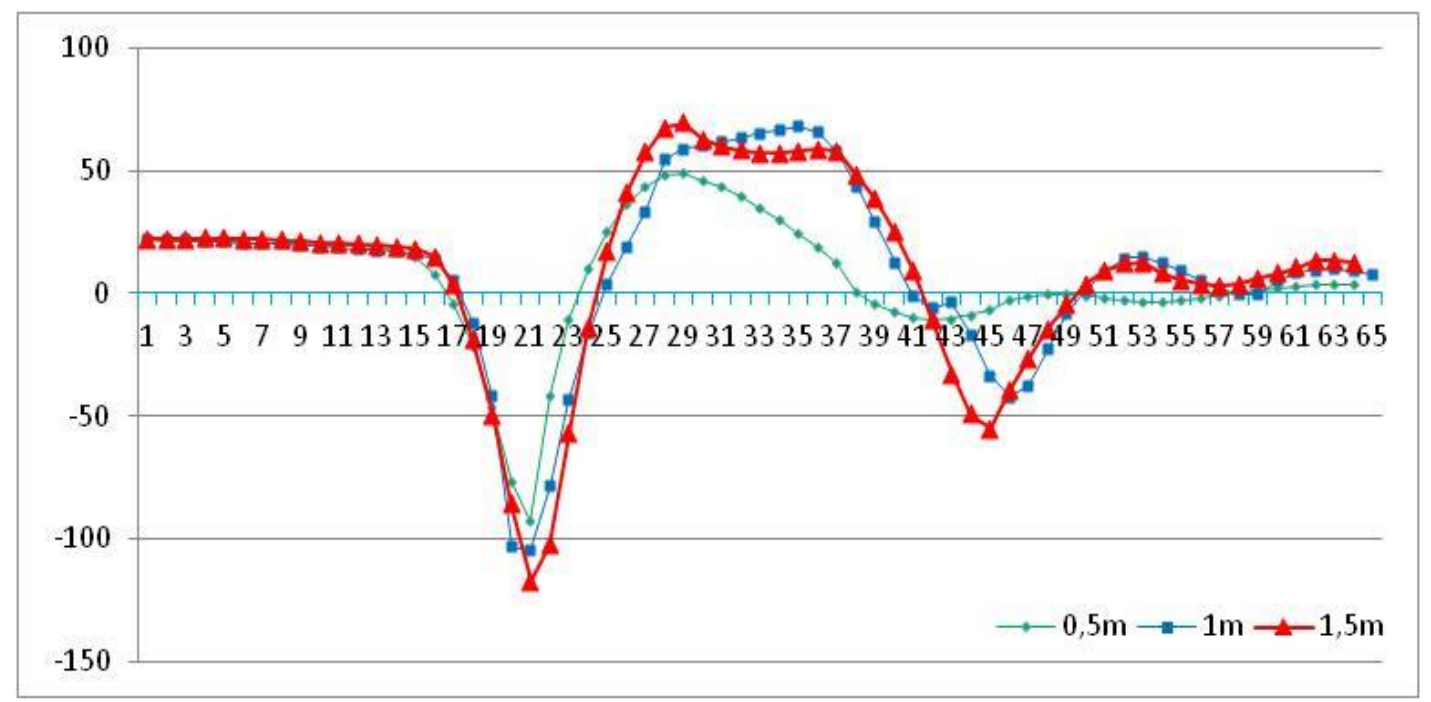

Figure 2. Values of acceleration in heading, nonfiltered data, sagittal axis

The results show that potential risks of head injuries may arise when the velocity of the flying ball is faster than the velocity simulated in this experiment. In spite of the slow speed, the maximum acceleration of the head reaches the value of up to $-123,037 \mathrm{~m} / \mathrm{s}^{2}$. The sensitivity of the device enabled to find out the time of the head-ball contact, which took up to $5 \mathrm{~ms}$. The results of the experiment conducted in the U11 age category (n 20) were presented at the conference in Szombathely in 2014.

\section{Karate}

The tested people were 2 men. The first one aged 30 with the black belt and the other one aged 12 with the yellow belt. $A$ basic stroke gyaku-cuki was performed. The accelerometer was firmly fixed on the inner part of the wrist, just above the palm so as not to move. The axis $x$ was parallel with the forearm and it measured the acceleration in the direction of the stroke. The axis $x$ also measured the rotation. The stroke was performed without hitting any concrete target. When the stroke is performed correctly, the maximum acceleration is involved at the end of the stroke, the duration of the stroke performance takes the shortest time period possible, and the rotation is involved during the last phase of the stroke. 


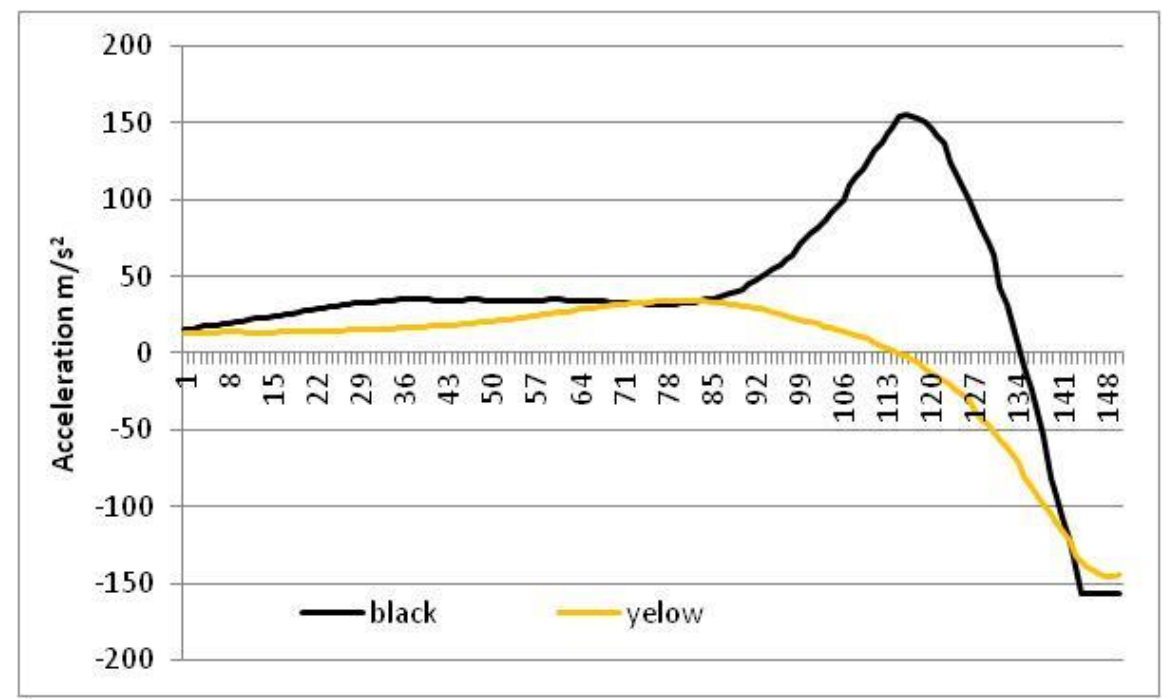

Figure 3. Acceleration during the stroke, nonfiltered data, black and yellow belt

Figure 3 depicts the course of the arm acceleration in the stroke gyaku-cuki. The phase takes $0.15 \mathrm{~s}$, which is the duration of the stroke performance in the tested person with the yellow belt. Positive values show the forward acceleration of the arm, the minus values show the stop of the arm at the end of the stroke. The duration of the stroke performance, the values of the maximum acceleration and the duration of the stroke stop can be gained and calculated from the data. In the person with the yellow belt, the duration of the acceleration takes longer, and the maximum acceleration is $34.1 \mathrm{~m} / \mathrm{s} 2$. The maximum acceleration is 154.9 $\mathrm{m} / \mathrm{s} 2$ in the person with the black belt. The performances of the stroke differ significantly. The performance of the person with the black belt is highly accurate.

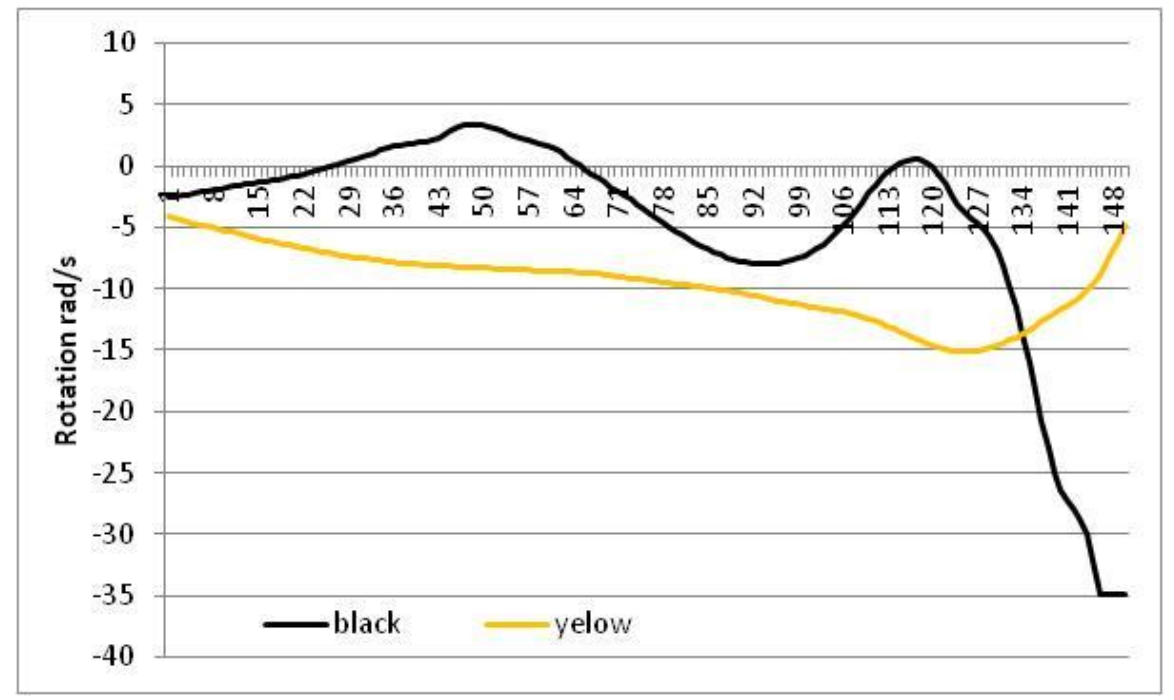

Figure 4. Wrist rotation of the stroke, nonfiltered data, black and yellow belt

Figure 4 shows the rotation of the wrist at the end of the performed stroke. The rotation was performed at the end of the stroke and the values of the maximum rotation are high, more than 35 radians per second in the person with the black belt. In the person with the yellow belt, the rotation began at the beginning of the stroke 
and was performed during the whole duration of the stroke, and the values of maximum rotation were lower, up to 15 radians per second. The minus values indicate the inward rotation of the hand.

\section{Shot Putting}

The tested person was an athlete doing decathlon. The accelerometer was fixed on his wrist. The results of shot putting from the standing position without any preparatory movement are shown in figure 5.

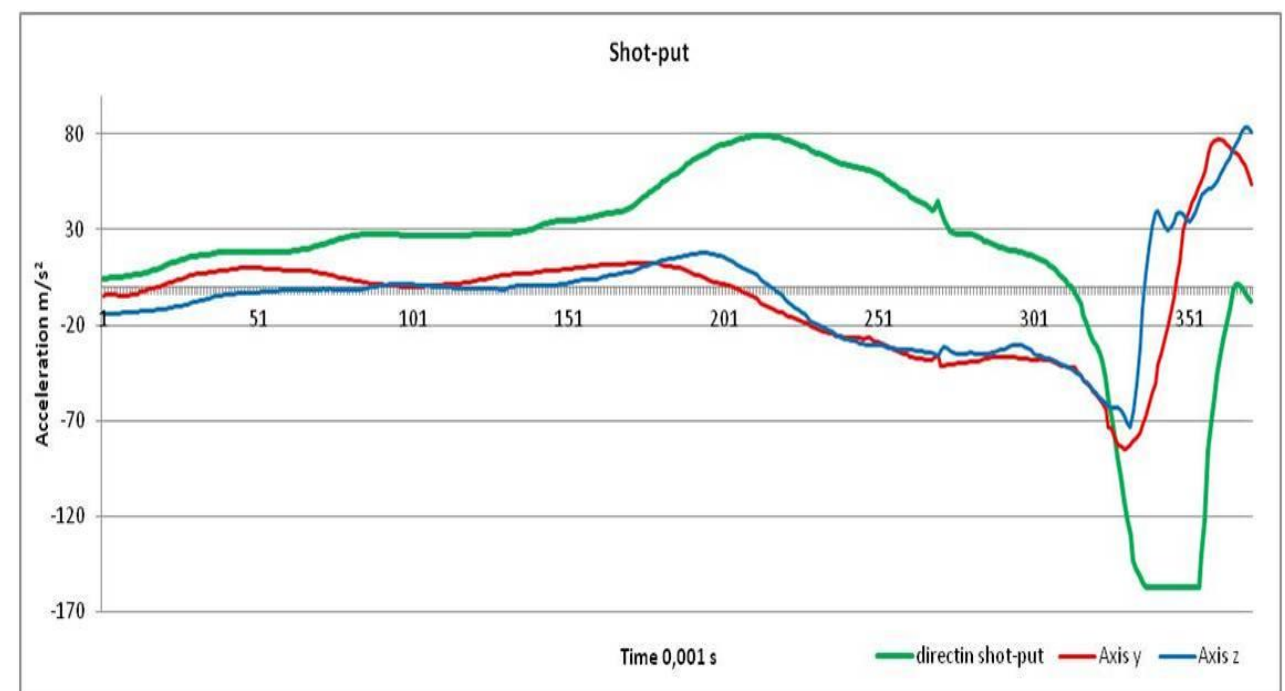

Figure 5. Shot putting from the standing position, filtered data

If performed accurately, the acceleration only appears at the axis $x$, and the acceleration at the axes $y$ and $z$ is involved after the ball leaves the hand. The acceleration curves in figure 5 show that the technique is imperfect. The forearm moved not only forward (the green curve), but also inward and downward (the red and blue curves).

\section{Cross-country Skiing}

The tested person was a man aged 50, a skiing lecturer. He had an operation in his right knee shortly before the experiment was conducted. The classical technique of diagonal stride was performed. The accelerometer was fixed on the low back to be as close to the centre of gravity of the sportsperson as possible. 


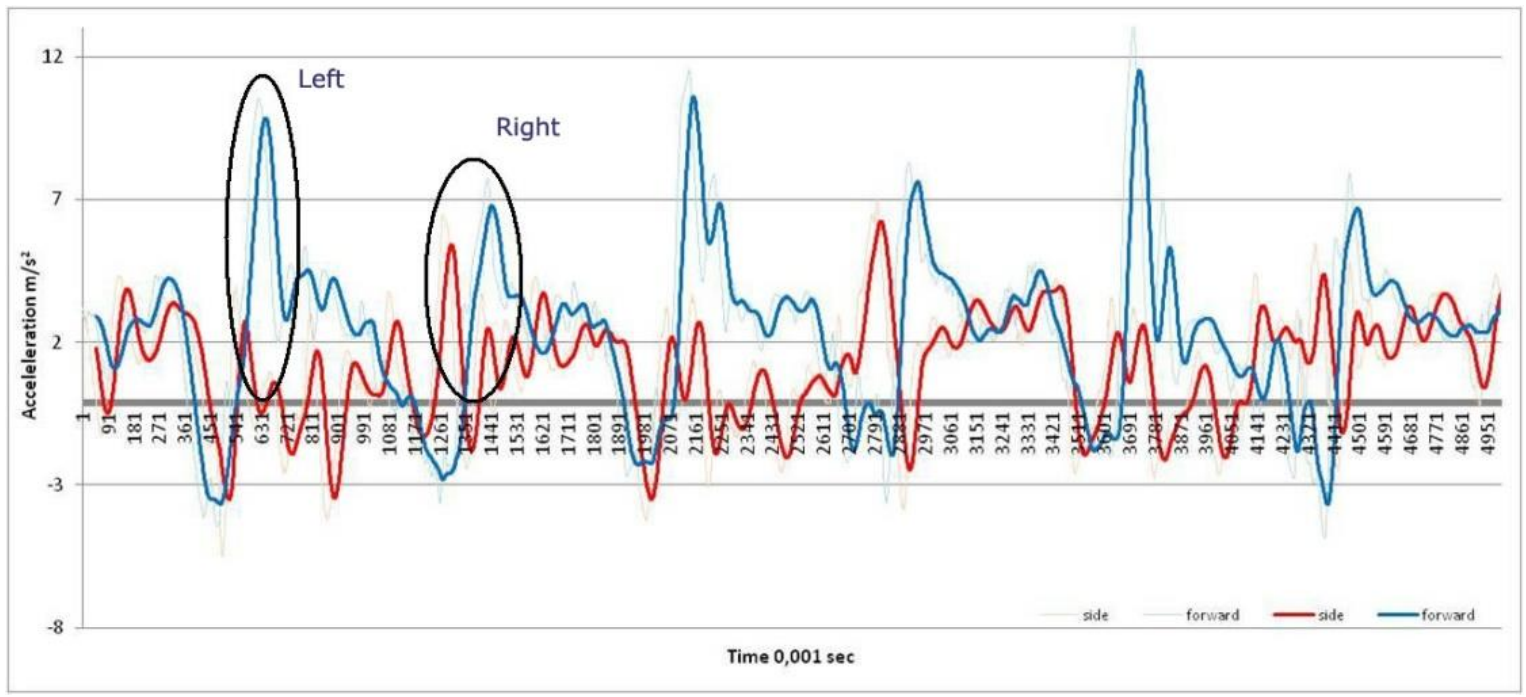

Figure 6. Cross-country skiing, classical technique, diagonal stride

Figure 6 reveals a wrong technique of the push-off phase in the right leg. The skier transfers the weight before the push-off, and thus the push-off is performed from the lightweight ski. The push-off is less dynamic in the right leg.

\section{Vertical Jump}

The tested person was a woman aged 30 , without any sports specialization. The accelerometer was fixed on the low back to be as close to the centre of gravity of the sportsperson as possible.

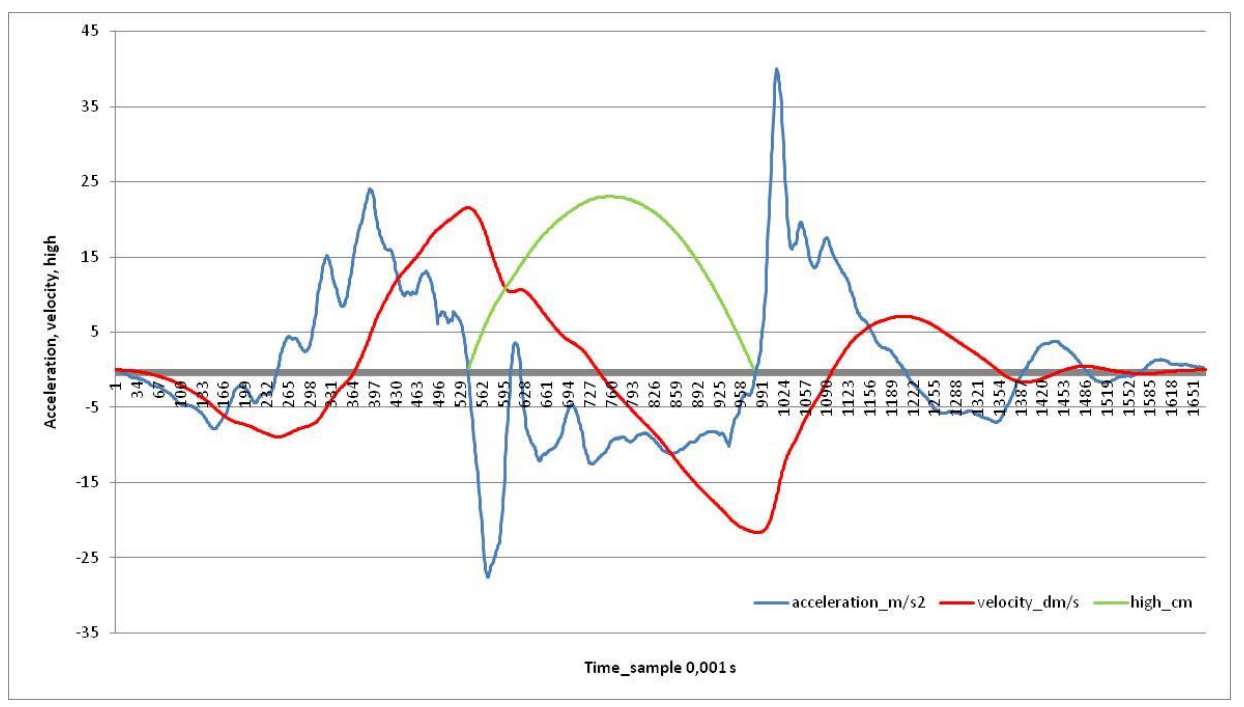

Figure 7. Vertical jump from standing position; acceleration, velocity and trajectory of the centre of gravity

Figure 7 illustrates the possibilities to use the accelerometer in jumping from the standing position. The velocity and trajectory of the jump can be calculated and designed from the gained data. The method can be also used for field measurements to monitor the fitness, fatigue and efficiency in repeated jumping. From the 
shape of the curve, the involvement of particular muscle groups in the jump can also be deducted and deduced.

\section{Skiff}

The tested person was a woman, a junior representative. The experiment was conducted in her transitional training period. The accelerometer was placed in a horizontal position on the skiff hull behind the rower's back.
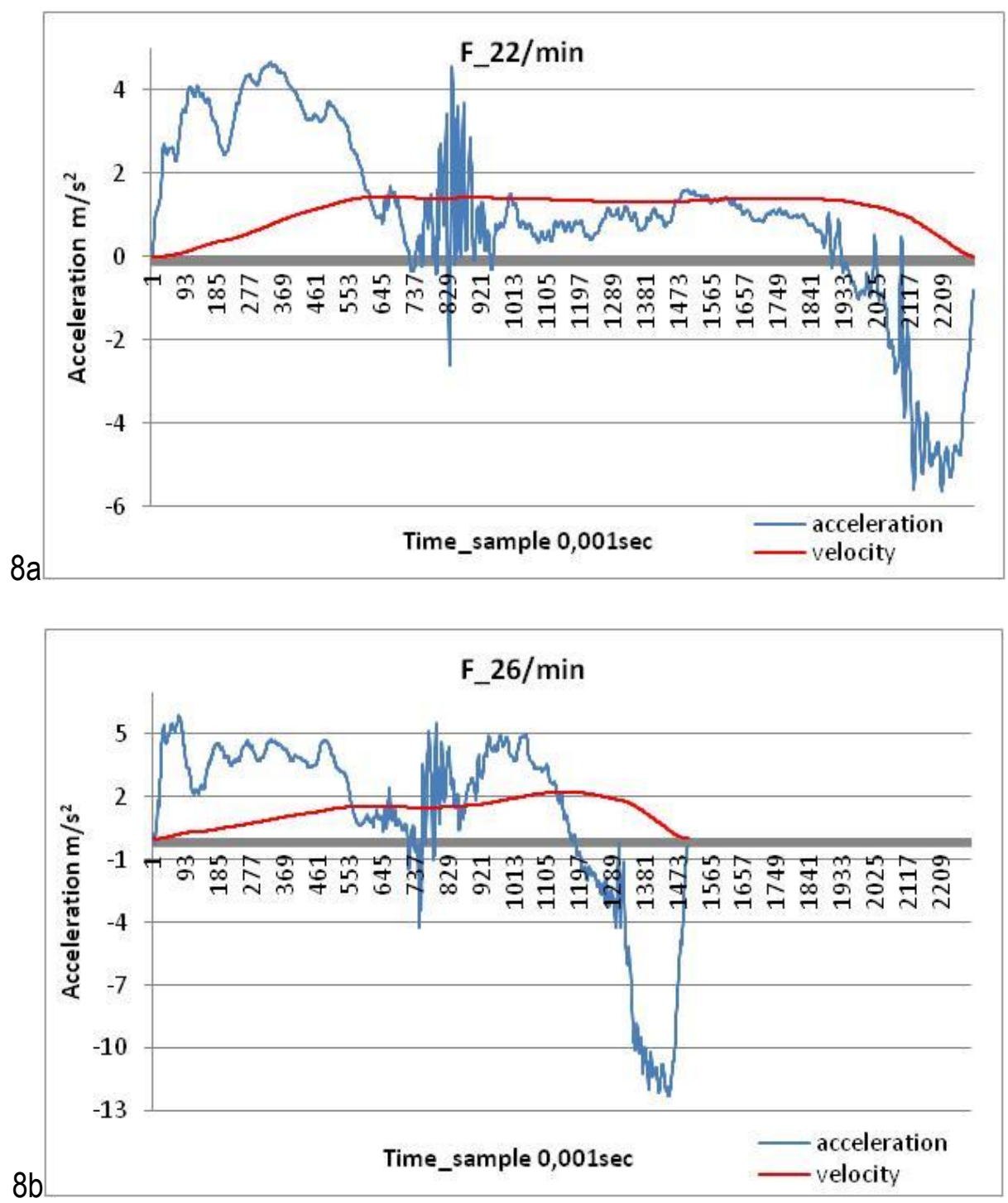

Figure 8. Acceleration and velocity, frequencies of 22 (a) and 26 (b) strokes per minute

From the curves in figures 8, the stroke phase, as well as the phase of the oars taking out of water and their above water transfer can be analysed. Regarding these two different stroke frequencies (22 and 26 stokes per minute), a significant difference appears at the beginning of the stroke phase. The involvement of the arm, back and leg muscle work differ, the maximum accelerations also vary (4 m/s $\mathrm{s}^{2}$ at the frequency of 22 strokes $/ \mathrm{min}$ and $5.4 \mathrm{~m} / \mathrm{s}^{2}$ at the frequency of 26 strokes $\left./ \mathrm{min}\right)$. The oar transfer phase is also significantly different. When the rower's seat is drawn closer, the skiff accelerates markedly. This is detectable from the 
curves of the skiff velocity in figure 9 (the calculation of the acceleration integration). The velocity values are not real; they show the velocity oscillation above the minimum velocity of the skiff $(3.0-3.3 \mathrm{~m} / \mathrm{s}$, the average velocity at the frequency of 22 strokes $/ \mathrm{min}$ is $4.1 \mathrm{~m} / \mathrm{s}$, and $4.3 \mathrm{~m} / \mathrm{s}$ at the frequency of $27 \mathrm{strokes} / \mathrm{min}$ ).

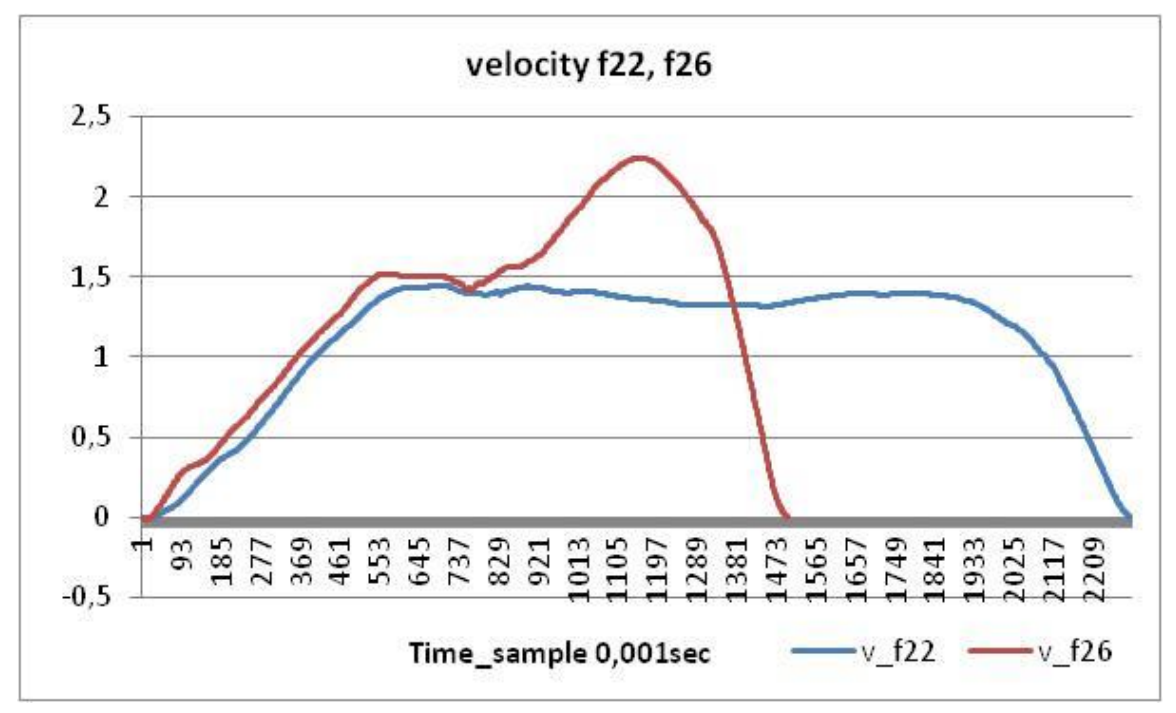

Figure 9 . Velocity of the skiff at frequencies of 22 and 26 strokes per minute

These analyses can become important aid tools for trainers when assessing rowing techniques and strength abilities and their involvement in rowing.

\section{Ski jumping}

The tested person was a boy, aged 17, a junior representative in ski jumping on the artificial ski jump K-70, the ramp velocity of $87-88 \mathrm{~km} / \mathrm{h}$. The accelerometer was fixed on the tested person's chest. The curve shows the vertical velocity of the torso straightening when taking-off. The gained data was compared with the data obtained from the dynamic platform located at the end of the ski jump, in the area where jumpers take off. 


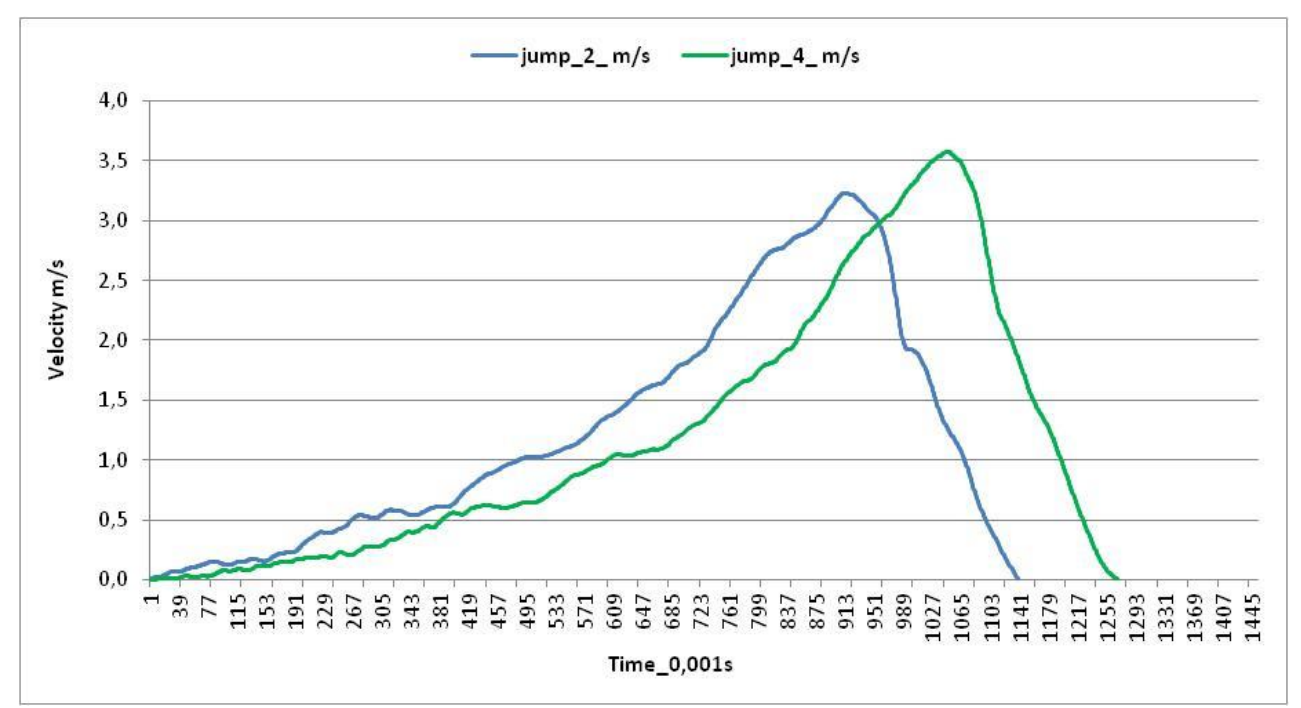

Figure 10. Vertical velocity of the torso in two ski jumps

Figure 10 shows that jump 2, the blue curve, was $1.5 \mathrm{~m}$ longer, although the values of the torso velocity when taking off are lower. The experiment was conducted repeatedly and the data analysis indicates that the jump length is not determined by the way of the take-off only. Many other factors play an important role here, among which the ski jumper flying skills are of significant importance.

\section{DISCUSSION}

Based on the data and analyses, provided by our experiments conducted in selected sports, we came to the following conclusions. Firstly, the placement of the accelerometer should be carefully considered, as stated by Atallah, et al. (2010). Since we only had one accelerometer available, its placement sometimes seemed to be troublesome. In ski jumping e.g., two accelerometers were placed (Chardonnens, et al., 2013); one on the ski jumper and the other one on the ski. Their findings showed statistically significant correlation relations between the length of the jump itself and the ski acceleration perpendicular to the ski jump. Regarding the movement and dynamics of the examined sportspeople, the findings do not provide any conclusions however. Our experiment reconfirmed that ski jumping is a multi-factor discipline, and the performance cannot only be described by one parameter.

When placing accelerometers on a tested body, the orientation of the axes towards the body is vital to record, to show clearly whether minus and plus values represent the forward and downward movement, potentially the movement to the sides. To record, to film or photograph, the position of the accelerometer axes towards the space and towards the direction of the performed movement is also desirable (Mathie, et al., 2004).

If the acceleration data is only used to analyse the movement activity and no other calculations are pursued, the following facts are essential to be taken into consideration: in vertical movements (jumps), the acceleration of gravity must be subtracted from the obtained data so that the acquired data can be relevant. The placement of the accelerometer is also crucial to gain relevant data (Elvin, et al., 2013). If shot putting is analysed, it is necessary to consider the data contamination (Songa, et al., 2006). Considering the fact that the throw of the ball is performed under the angle of $45^{\circ}$, the acceleration of gravity is split between two axes. Since the angle of the ball throw is usually standard in throwers, the data can be analysed and compared 
without complex modifications. Considering the sensitivity of accelerometers, accelerometry is a suitable method to analyse short-lasting movements, strokes and shocks (Lukášek and Kalichová 2014). In longlasting cyclic sports (rowing, running, swimming, cross-country skiing, walking etc.), the accelerometry data is also helpful and beneficial, as separate cycles can be analysed. Subsequently, the impact of the fatigue, fitness and efficiency on sports performances and techniques can be inferred and concluded (Kavanagh, et al., 2008).

If other calculations are pursued from the data obtained by accelerometers (e.g. velocities, trajectories), the following aspects should be taken into consideration: In the case of a linear motion in a plane without significant tilts of the object in the vertical and horizontal direction (rowing), several-second records can be accepted. In longer-lasting performances the accuracy decreases significantly, as the contamination and errors increase even in these relatively linear motions. In cyclical movements, velocities and trajectories are advisable to calculate from particular cycles, as it is considerably more accurate. In the case of movements along a straight line which are accompanied by movements in horizontal and vertical directions (swimming, running), the contamination and errors increase significantly, e.g. in swimming up to $12-15 \%$ (Stam, et al., 2011). In the case of walking, the data of the acceleration and rotation can be used separately, thus the calculations do not involve any significant contamination and errors (Kavanagh, et al., 2008). To analyse movements performed multi-directionally, in which movements along the inclined plane are included, the calculations are rather inaccurate, and we declare that the acceleration and rotation data can be only used. Based on our measures and findings, we conclude that accelerometry is optimal and appropriate to use for the analysis of dynamic, relatively simple movements performed along standard, anticipated and optimum trajectories, such as in vertical jumping, springing, strokes, shocks and impacts, and to assess overloading of joints, e.g. in long jumps or triple jumps.

\section{CONCLUSIONS}

Regarding the findings provided in our experiments, we conclude that the method of accelerometry is suitable and convenient for movement analyses in all sports, where acceleration values are important parameters of the technique and dynamics of the performed sports movements. To understand and analyse the obtained data properly, the results provided by gyroscopes, recording the data about rotations of the measured segments, are supportive and contributory. All the data can also show changes in the dynamics of a movement in difficult or changing conditions, or the ones caused by fatigue.

In future research, we propose to focus on the involvement of more accelerometers to measure sports movement activities. The remote data transfers are desirable to involve, and more sophisticated software for the data assessment should be developed.

\section{REFERENCES}

1. Atallah, L., Lo, B., King, R., \& Yang, G. Z. (2010). Sensor Placement for Activity Detection Using Wearable Accelerometers. Proceeding BSN '10 Proceedings of the 2010 International Conference on Body Sensor Networks, 24-29.

2. Bampouras, T. M., Relph, N. S. Orme, D., \& Esformes, J.I. (2013). Validity and reliability of the Myotest Pro wireless accelerometer in squat jumps. Isokinetics and Exercise Science, 21(2), 101105.

3. Bernmarka, E., \& Wiktorina, Ch. (2002). A triaxial accelerometer for measuring arm movements. Applied Ergonomics, 33(6), 541-547. 
4. Bogert van den, A. J., Read, L., \& Nigg, B. M. (1996). A method forcinverse dynamic analysis using accelerometry. Journal of Biomechanics, 29(7), 949-954.

5. Elvin, N. G., Elvin, A. A., \& Arnoczky, S. P. (2007). Correlation between Ground Reaction Force and Tibial Acceleration in Vertical Jumping. Journal of Applied Biomechanics, 23(3), 180-189.

6. Hamill J., \& Knutzen K. (2003). Biomechanical basis of human movement. Baltimore: Lippincott Williams \& Wilkins, 2nd edition.

7. Chardonnens, J., Favre, J., Cuendet, F., \& Aminian, K. Measurement of the dynamics in ski jumping using a wearable inertial sensor-based system. Journal of Sports Sciences, 2013.

8. Kavanagh, J. J., Hylton, B., \& Menz, H. B. (2008). Accelerometry: A technique for quantifying movement patterns during walking. Gait \& Posture, 28(1), 1-15.

9. Liu, J., Wang, J., Zhao, Y., Zhao, Q., Li, J., \& Dongxue, C. (2011). Research on the Application of the Three-dimensional Information Collection System of Digital Discus to the Throwing Technique of the Discus Throwers. International Conference on Future Computer Science and Education, 196199.

10. Lukášek, M., Kalichová, M. (2014) Injury Risks of Heading in Young Football Players. The 9th INSHS International Christmas Sport Scientific Conference "Qualitative and Quantitative Research in Sport Science", Szombathely, Hungary.

11. Mathie, M. J., Coster, A. C. F., Lovell, N. H., \& Celler, B. G. (2004). Accelerometry: providing an integrated, practical method for long-term, ambulatory monitoring of human movement. Physiol Meas, 25, 20-38.

12. Mayagoitia, R. E., Nene, A. V., \& Veltink, P. H. (2002). Accelerometer and rate gyroscope measurement of kinematics: an inexpensive alternative to optical motion analysis systems. Journal of Biomechanics, 35, 537-542.

13. Mizrahi, J., Verbitsky, O., Isakov, E., \& Daily, D. (2000). Effect of fatigue on leg kinematics and impact acceleration in long distance running. Human Movement Science, 19(2), 139-151.

14. Nakashima, M., Ohgi, Y., Akiyama, E., \& Kazami, N. (2010). Development of a swimming motion display system for athlete swimmers' training using a wristwatch-style acceleration and gyroscopic sensor device. Procedia Engineering, 2(2), 3035-3040.

15. Naunheim, R. S., Bayly, P. V., Standeven, J., Neubauer, J. S., Lewis, L. M., \& Genin, G. M. (2003). Linear and angular accelerations during heading of a soccer ball. Medicine and Science in Sports and Exercise, 35(8), 1406-1412.

16. Sell, T. C., Akins, J. S., Opp, A. R., \& Lephart, S. M. (2014). Relationship Between Tibial Acceleration and Proximal Anterior Tibia Shear Force Across Increasing Jump Distance. Journal of Applied Biomechanics, 30(1), 75-81.

17. Songa, G., Yuan, H., Tangc, Y., Songd, Q., \& Ged, Y. (2006). A novel three-axis force sensor for advanced training of shot-put athletes. Sensors and Actuators, 128(1), 60-65.

18. Stamm, D., James, J., \& Thiel, V. (2013). Velocity profiling using inertial sensors for freestyle swimming. Sports Eng., 16(1), 1-11.

19. Stamm, A., Thiel, D. V., Burket, B., \& James, D. A. (2011). Towards determining absolute velocity of freestyle swimming using 3-axis accelerometer. Procedia Engineering, 13, 120-125.

20. Veltink, P. H., Bussmann, H. B., de Vries, W., Martens, W. L., \& Van Lummel, R. C. (1996). Detection of static and dynamic activities using uniaxial accelerometers. IEEE Trans Rehabil Eng., 4(4), 375385. 